\title{
Ultrasound assessment of normal hemidiaphragmatic movement: relation to inspiratory volume
}

\author{
J G Houston, R M Angus, M D Cowan, N C McMillan, N C Thomson
}

\begin{abstract}
Background - Traditionally the radiological assessment of diaphragmatic movement has relied on fluoroscopy. Ultrasound scanning has recently been shown to be a sensitive and reproducible method of assessing hemidiaphragmatic movement in normal subjects. A study was undertaken to examine how movement of the diaphragm measured by ultrasound scanning relates to inspired lung volumes measured by spirometric testing.
\end{abstract}

Methods - Ultrasound examinations were performed on 14 normal volunteers using a $3.5 \mathrm{MHz}$ sector transducer (Acuson 128). A fixed skin position on each lateral chest wall between the anterior clavicular and midaxillary line was selected symmetrically to obtain a longitudinal plane of each hemidiaphragm including the maximal renal bipolar length, allowing identification of the adjacent posterior aspect of the diaphragm. Craniocaudal excursions of the posterior part of each hemidiaphragm on successive respiratory cycles were recorded on videotape and compared with spirometric measurements recorded simultaneously on a water bath spirometer. Measurements were made in the sitting and supine position and were repeated on a separate occasion (at least two weeks apart) in 10 subjects by the same operator to assess reproducibility. Results - The relation between inspired volume and hemidiaphragmatic movement was found to be linear. The gradient of these observed linear relations (hemidiaphragmatic excursion (mm)/inspired volume (1)) was calculated and their distribution for each hemidiaphragm followed a normal distribution irrespective of position. The $95 \%$ confidence limits of the right to left ratio of these gradients in the supine position were 0.53 and 1.7 . Change of posture from the supine to the sitting position reduced the gradient. The technique had acceptable reproducibility with coefficients of variation for the supine position of $7.5 \%$ and $11.7 \%$ for right and left hemidiaphragm respectively.

Conclusion - Ultrasound scanning is a simple, non-invasive and reproducible means of assessing hemidiaphragmatic movement, yielding quantitative in- formation which relates to inspired lung volumes.

(Thorax 1994;49:500-503)

The radiological assessment of diaphragmatic function has traditionally relied upon plain radiography and erect fluoroscopy. 'Information about hemidiaphragmatic movement from plain radiography is limited because of the wide normal variability of hemidiaphragmatic position, ${ }^{2}$ while fluoroscopy is complicated both by conflicting reports of side to side variability ${ }^{23}$ and an apparent $6 \%$ incidence of paradoxical movement on sniffing in normal subjects. ${ }^{3}$ Ultrasound scanning is an accepted qualitative method of assessing hemidiaphragms - for example, subpulmonary effusions, subphrenic collections ${ }^{1}$ and, more recently, traumatic rupture ${ }^{45}$ and pleural masses. ${ }^{6}$ We have evaluated ultrasound scanning as a quantitative method of assessing hemidiaphragmatic movement in normal subjects. $^{7}$ The normal ranges of craniocaudal excursion of the posterior hemidiaphragm (both in tidal and maximum voluntary respiration), and the right to left variability were determined, and acceptable reliability of the technique in terms of the interobserver and intraobserver reproducibility was shown.

Using erect fluoroscopy it has been shown that the anterior part of the hemidiaphragm moves approximately $40 \%$ less than the posterior part, and that the mean axial motion is linearly related to the volume displacement of the diaphragm as measured by both respiratory induction plethysmography (Respitrace) and fluoroscopy in the erect position. ${ }^{8}$ This conflicts with the results of a study of the diaphragm using magnetic resonance imaging (MRI) in the supine position. ${ }^{9}$

The aims of this study were to determine the relation of the posterior diaphragmatic excursion and inspired volume as measured by simultaneous ultrasound scanning and waterbath spirometric testing, to examine the variability of this relation in a normal group of subjects, to observe any difference in changing posture, and to assess the reproducibility of the relationship.

\section{Methods}

SUBJECTS

Fourteen healthy subjects with a wide range in age and body habitus (table 1 ), unpractised in 
performing respiratory manoeuvres, were studied. The age, height, weight, and chest circumference of each were recorded. In addition maximum and average peak inspiratory and expiratory mouth pressures, both in a supine and sitting position, were recorded as the best of three trials using a dedicated mouth pressure monitor (Chest Scientific).

\section{STUDY DESIGN}

Baseline ultrasound assessment of each hemidiaphragm was performed in the supine and sitting positions. Each subject was then simultaneously assessed with ultrasound while settled in a pattern of tidal breathing on a waterbath spirometer, both in the supine and sitting position. A series of inspired volumes was performed over the range from functional residual capacity to vital capacity. Spirometric data were recorded on the tracing and the diaphragmatic excursion recorded as before, the tape and trace being annotated to allow subsequent correlation for each breath. To assess reproducibility the study was repeated on a separate occasion (at least two weeks apart) in 10 subjects by the same operator.

\section{ULTRASOUND EXAMINATION}

Ultrasound examinations were performed with a $3.5 \mathrm{MHz}$ sector transducer (Acuson 128). A fixed skin position on each lateral chest wall between the anterior clavicular and midaxillary line was selected symmetrically to obtain a longitudinal plane of the hemidiaphragm including the maximal renal bipolar length, allowing identification of the adjacent posterior aspect of the diaphragm. Craniocaudal excursions on successive respiratory cycles were recorded on videotape (Super VHS). The excursion was later analysed independently by placing a craniocaudal displacement line at the depth of the midpoint of the kidney on expiration and the excursion on inspiration measured as on fig 1 .

\section{SPIROMETRIC TESTS}

Continuous spirometric measurements were made with a water seal spirometer (Gould Pulmonet 3).

Table 1 Subject characteristics

\begin{tabular}{lllll}
\hline Sex & $\begin{array}{l}\text { Age } \\
\text { (years) }\end{array}$ & $\begin{array}{l}\text { Height } \\
\text { (cm) }\end{array}$ & $\begin{array}{l}\text { Weight } \\
\text { (kg) }\end{array}$ & $\begin{array}{l}\text { Chest } \\
\text { circumference } \\
\text { (cm) }\end{array}$ \\
\hline M & 31 & 182 & 90 & 130 \\
M & 29 & 183 & 73 & 100 \\
M & 26 & 198 & 99 & 127 \\
M & 23 & 165 & 76 & 105 \\
M & 29 & 187 & 87 & 102 \\
M & 25 & 188 & 76 & 95 \\
M & 55 & 180 & 69 & 86 \\
F & 40 & 175 & 71 & 98 \\
F & 24 & 175 & 73 & 95 \\
F & 30 & 165 & 63 & 92 \\
F & 69 & 167 & 56 & 83 \\
\hline
\end{tabular}

\section{STATISTICAL ANALYSIS}

Data analysis was performed on an IBM PC2 using the Minitab software package. This involved use of the "goodness of fit technique" to assess normality (summarised as mean, standard deviation, and standard error of the mean), simple linear regression analysis to assess scatter, parametric tests such as Student's $t$ test and analysis of variance to assess reproducibility. In particular, the relation between posterior hemidiaphragmatic excursion for sequential respiratory cycles and the inspired volume of that cycle was examined and described in terms of its gradient (hemidiaphragmatic excursion ( $\mathrm{mm})$ /inspired volume (1)), and intercept.

The variation of these parameters within the individuals, both supine and sitting, was compared and the possible factors determining the gradient (height, weight, age, mouth pressures, and chest circumference) in this small group of individuals analysed to assess whether this relation might be predicted from these variables in any individual.

\section{Results}

All subjects tolerated the study and there were no withdrawals. Peak mouth pressures were in keeping with normal diaphragmatic function (table 2) making major weakness unlikely. In all subjects adequate views of each hemidiaphragm over the range of inspiratory volumes was obtained. It was noted that subjects with large excursions required careful attention to ensure full visualisation of each end of the respiratory cycle unobscured by the underlying movement of the ribs.

The relation between inspired volume and hemidiaphragmatic excursion was plotted and in all cases was seen to be linear (example of one subject fig 2 ) with little scatter, the mean (range) $R^{2}$ values (\%) being 96 (89-99) and 96 (87-99) for right and left respectively in the supine position, and 94 (83-99) and 95 (87-99) in the sitting position. The gradients of these observed linear relations were calculated (hemidiaphragmatic excursion $(\mathrm{mm})$ /inspired volume (1)) and the distribution and range of these gradients for each hemidiaphragm in each position was plotted and noted to be of normal distribution (table 3, fig 3). The mean (SD) of each hemidiaphragm in the same position was not significantly different. The mean (SD) right to left ratio of the gradients was $1.02(0.18)(95 \%$ CI 0.66 to 1.4$)$ for the supine position and $1.13(0.29)(95 \%$ CI 0.53 to 1.7$)$ for the sitting position.

To evaluate the variation of this relation of inspired volume to diaphragmatic excursion in this small group the gradients for each subject for each hemidiaphragm in each position were compared with height, weight, age, mouth pressures, and chest circumference. No clear relation was found with a single variable. The best predictors of the gradient were, in decreasing order of power: vital capacity, weight, peak maximal and average expiratory mouth pressure. There was a significant postural difference in the gradients in a subject which 
A
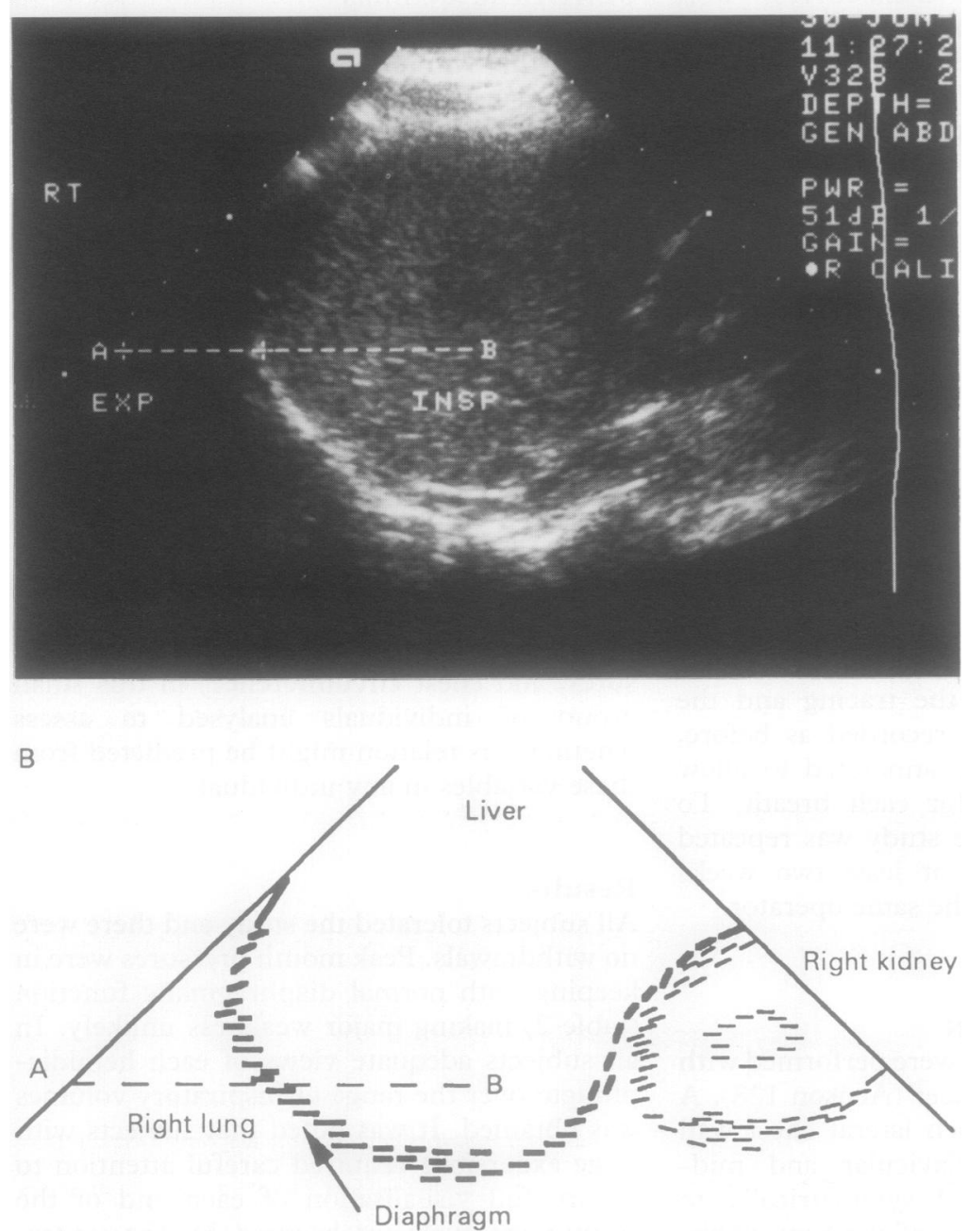

Figure 1 (A) Ultrasound scan showing measurement of the craniocaudal excursion on inspiration of the posterior left hemidiaphragm. (B) Line diagram showing measurement of the craniocaudal excursion on inspiration of the posterior left hemidiaphragm.
Table 2 Mean ( $S E$ ) peak mouth pressures $\left(\mathrm{Cm} \mathrm{H}_{2} \mathrm{O}\right)$

\begin{tabular}{llc}
\hline & $\begin{array}{l}\text { Men } \\
(n=10)\end{array}$ & $\begin{array}{l}\text { Women } \\
(n=4)\end{array}$ \\
\hline Supine peak inspiratory pressure & $102(6 \cdot 10)$ & $87(10 \cdot 0)$ \\
Supine peak expiratory pressure & $118(8 \cdot 25)$ & $88(3.2)$ \\
Sitting peak inspiratory pressure & $112(6.0)$ & $99(8.5)$ \\
Sitting peak expiratory pressure & $126(8 \cdot 6)$ & $111(15.9)$ \\
\hline
\end{tabular}

Table 3 Distribution gradients for each hemidiaphragm and position (14 normal subjects)

\begin{tabular}{llll}
\hline Hemidiaphragm & \multicolumn{3}{l}{ Gradient $(\mathrm{mm} / \mathrm{l})$} \\
\cline { 2 - 4 } & Mean & $S D$ & $S E$ \\
\hline Supine & & & \\
$\quad$ Right & 18.0 & 3.87 & 1.04 \\
$\quad$ Left & 18.0 & 4.45 & 1.19 \\
Sitting & & & \\
$\quad$ Right & $15 \cdot 1$ & 4.68 & 1.25 \\
Left & 13.7 & 3.40 & 0.91 \\
\hline
\end{tabular}

tended to decrease on moving from the supine to the sitting position (fig 4 , right $\mathrm{p}<0.05$, left $\mathrm{p}<0.01$ ).

Intraobserver reproducibility was better in the supine position with a coefficient of variance for the right hemidiaphragm of $7.5 \%$ compared with $11 \cdot 7 \%$ on the left. This compared favourably with the sitting position with $22 \%$ on the right and $18 \%$ on the left.

\section{Discussion}

In accordance with our previous study ${ }^{7}$ ultrasound examination proved a simple, well tolerated, reproducible method of assessing hemidiaphragmatic movement in normal subjects. Its advantages over fluoroscopy are lack of risk from ionising radiation, portability, and direct quantitative information of the greatest amplitude of hemidiaphragmatic movement. The relation of this observed excursion with a range of inspired volumes appears linear for each hemidiaphragm in both the supine and sitting position. In this small group of normal subjects the range of gradients of these linear relations is not significantly different, and the range of the ratio of right to left gradients in the supine position $(0.66-1.4)$ is similar to that of the observed ratio of right to left excursions on maximal voluntary ventilation in our previous study using ultrasound assessment alone $(0 \cdot 5-1 \cdot 6)$. This supports the observation that there is a wide range of side to side variability in hemidiaphragmatic movement in normal subjects, but this can be evaluated with ultrasound. It is interesting to note that this is in agreement with the fluoroscopic "rule of thumb" described by Alexander" that "the unequal movement of the two leaves of the diaphragm is usual, and unlikely to be of significance unless one excursion is at least twice as great as the other."

It is well known that vital capacity tends to rise on moving from a supine to an erect posture. ${ }^{10}$ In addition, the positions of the hemidiaphragms on an inspiratory chest radiograph are known to be higher in a supine radiograph than one taken in the erect posi-

Figure 2 Relation of hemidiaphragmatic excursion and inspired volume in one subject. 


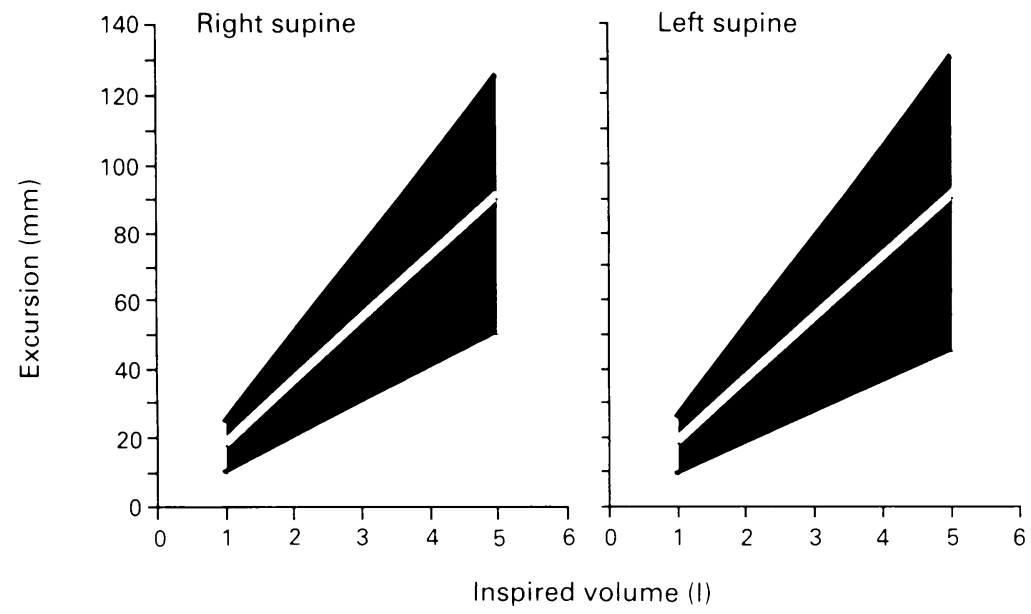

Figure 3 Range of ariability in gradient in each hemidiaphragm $95^{\circ}$ o confidence limits.
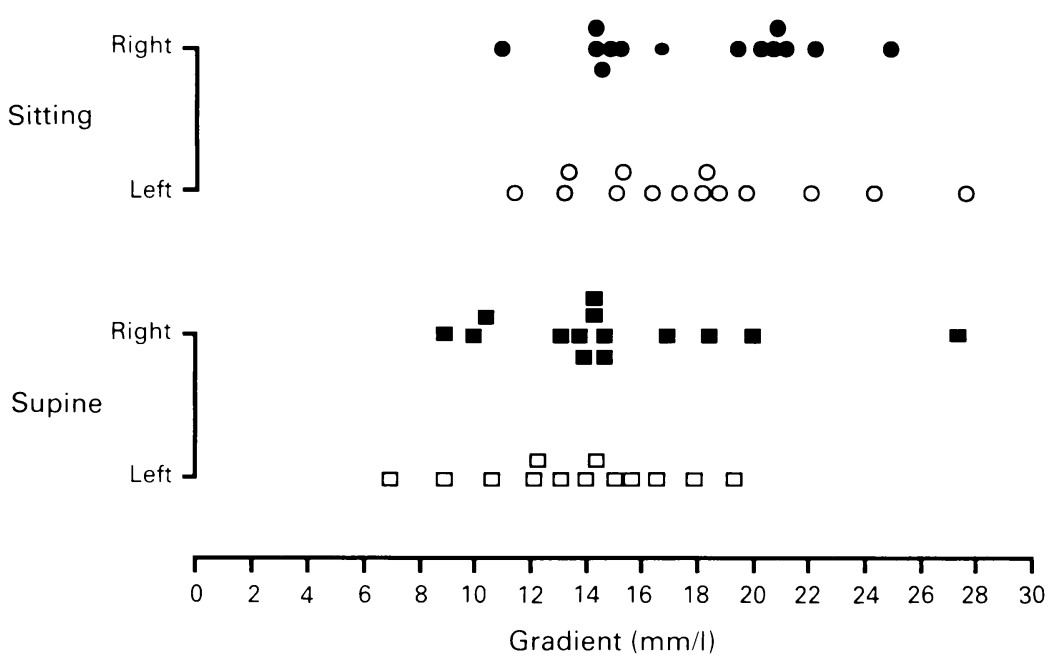

Figure 4 Siatter plot showing the effect of posture on gradient.

tion. "We observed a significant fall in the gradient $(\mathrm{mm} \mathrm{l})$ on this change of posture, implying a greater diaphragmatic excursion for the same volume inspired in the supine position.

In terms of the reproducibility of these observations, expressed as intraobserver variability, the coefficients for the supine position of $10 \%$ and $11 \%$ for right and left hemidiaphragms respectively imply acceptable reliability for serial testing, although less favourable than ultrasound examination alone. ${ }^{-}$The supine position is preferred in the assessment of hemidiaphragmatic movement in terms of the variability in observations in one side, varia- bility in side to side variability and reproducibility, in keeping with previous work. ${ }^{12}{ }^{13}$

This study reaffirms the acceptability of ultrasound examination as a non-invasive method of quantitatively assessing hemidiaphragmatic movement and, in particular, can be used to determine the relation of posterior diaphragmatic excursion to inspired volume non-invasively.

Posterior hemidiaphragmatic excursion, assessed by ultrasound examination as we have described, appears to be directly proportional to inspired volume of that inspiratory cycle. The observed hemidiaphragmatic excursion variability between each hemidiaphragm in any individual must be considered in the investigation of suspected hemidiaphragmatic paresis or paralysis. Allowing for the range of variability in normals, this can be accurately assessed by ultrasound examination. Ultrasound examination also has advantages over traditional fluoroscopy in that it makes a direct quantitative assessment and is portable. It may therefore have potential use in the investigation of suspected hemidiaphragmatic paresis or paralysis.

The authors would like to thank Aileen Brown. Heather Ambler, and Rita Jack of the Respiratory Function Laboratory, Gartnavel General Hospital for their help with this study, and Catherine Howie of the Eniversity Department of Medicine and Therapeutics, Western Infirmary, Glasgow for her help with statistical analysis.

1 Flower CDR. The diaphagm: diagnostic radiology. Vol 1 Churchill Livingstone, 1986:170.

2 Simon G. Principles of chest x-rav diagnosis. London: Butterworths, 1956.

3 Alexander C. Diaphragmatic movements and the diagnosis of diaphragmatic paralysis. Clin Rudiol 1960:17:79-83.

+ Somers J.M. Gleeson FV. Flower CDR. Rupture of the right hemidiaphragm following blunt trauma: the use of right hemidiaphragm following blunt trauma: the use of
ultrasound in diagnosis. Clin Radiol 1990;42:97-101

5 Ammann A.M. Brewer WH, Maull KI, Walsh JW. Traumatic rupture of the diaphragm: real-time sonographic matic rupture of the diaphragm:
diagnosis. $A f R \quad 1983 ; 140: 915-6$.

6 Lipscomb DJ, Flower CDR, Hadfield JO. Lltrasound of the pleura: an assessment of clinical value. Clin Radiol 1981;32:289-90.

7 Houston JG, Morris AD, Howie CA, Reid JL, Mc.Millan $\therefore$. Technical report: quantitative assessment of hemidiaphragmatic movement. A reproducible method using ultrasound. Clin Radiol 1992:46:405-

8 Verschalaken JA, Deschepper K, Jiang TX, Demedts $M$ Diaphragmatic displacement measured by fluoroscopy and derived by Respitrace. F Appl Physiol 1989;67:694-8

9 Paiva M, Verbanck ?. Estenne M, Poncelet B, Segebarth C, Macklem PT. Mechanical implications of in vivo human diaphragmatic shape. F A ppl Physiol 1992;72:1407-12.

diaphragmatic shape. F App/ Phlstol $1992 ; 72: 1407-12$.
10 Allen S.M. Hunt B, Green M. Fall in vital capacity with posture. Br 7 Chest Dis 1985;79:267-72.

11 Wade OL, Gibson JC. The effect of posture on diaphragmatic movement and vital capacity in normal subjects with a note on spirometry as an aid in determining radiological chest volumes. Thorax 1951;6:103-26.

12 Loh L. Goldman M, Davis J. The assessment of diaphragmatic function. Hedicine 1977;60:170-2.

13 Moxham J. Tests of respiratory function. In: Problims in repiratory care Vol 3, vo 3,$1990 ; 313-20$ 